\title{
Square Diaphragm CMUT Capacitance Calculation Using a New Deflection Shape Function
}

\author{
Md Mosaddequr Rahman ${ }^{1}$ and Sazzadur Chowdhury ${ }^{2}$ \\ ${ }^{1}$ Department of Electrical and Electronic Engineering, BRAC University, 66 Mohakhali, Dhaka 1212, Bangladesh \\ ${ }^{2}$ Department of Electrical and Computer Engineering, University of Windsor, Windsor, ON, N9B 3P4, Canada
}

Correspondence should be addressed to Md Mosaddequr Rahman, mdmosaddeq@gmail.com

Received 8 June 2011; Accepted 21 July 2011

Academic Editor: Csaba Dücső

Copyright ( $) 2011$ M. M. Rahman and S. Chowdhury. This is an open access article distributed under the Creative Commons Attribution License, which permits unrestricted use, distribution, and reproduction in any medium, provided the original work is properly cited.

\begin{abstract}
A new highly accurate closed-form capacitance calculation model has been developed to calculate the capacitance of capacitive micromachined ultrasonic transducers (CMUTs) built with square diaphragms. The model has been developed by using a twodimensional polynomial function that more accurately predicts the deflection curve of a square diaphragm deformed under the influence of a uniform external pressure and also takes account of the fringing field capacitances. The model has been verified by comparing the model-predicted deflection profiles and capacitance values with experimental results published elsewhere and finite element analysis (FEA) carried out by the authors for different material properties, geometric specifications, and loading conditions. New model-calculated capacitance values are found to be in excellent agreement with published experimental results with a maximum deviation of $1.7 \%$, and a maximum deviation of $1.5 \%$ has been observed when compared with FEA results. The model can help in improving the accuracy of the design methodology of CMUT devices and other MEMS-based capacitive type sensors built with square diaphragms.
\end{abstract}

\section{Introduction}

Capacitive micromachined ultrasonic transducers (CMUTs) have become the choice of technology for a wide range of applications such as medical diagnostic imaging, nondestructive testing, material characterization, and automotive collision avoidance applications like park assist or blindspot monitoring. This type of sensors exhibit many advantages over their piezoelectric counterparts, such as inherently low power consumption, very high resolution and sensitivity, excellent stability and durability, lower sensitivity to temperature variations, low noise features, and monolithic and IC integration [1-3]. The typical CMUT geometry is built with a square, circular, or hexagonal diaphragm separated from a fixed backplate by a small airgap. When subjected to an incident ultrasonic wave, the diaphragm deforms and the capacitance between the diaphragm and the fixed backplate changes dynamically following the dynamic characteristics of the incident wave. A suitable microelectronic circuit is used to convert the capacitance change to a useful voltage signal [4].

As the CMUT's sensitivity depends on the change of capacitance, an accurate analytical method is necessary to calculate the capacitance between the deformed diaphragm and the backplate for any amount of deformation. However, as the diaphragm is rigidly clamped at the edges, the center of the diaphragm deflects more compared to the regions closer to the edges resulting in a cosine-like deformation curve. As one of the electrodes is curved, a parallel plate approximation to calculate the capacitance will introduce significant error. An accurate analytical approach to calculate the capacitance of such a geometry is to divide the curved electrode into a number of elemental areas where the elemental areas are small enough to be considered to form a parallel plate geometry with the flat electrode and then integrating over the length and width of the curved electrode to determine the total capacitance. Thus, accurate determination of the capacitance depends not only on the accuracy of the 
deflection of diaphragm center but also on the accuracy of the deformed shape of the diaphragm.

As the exact shape of a deflected clamped edge diaphragm is not known, generalized plate theory has been applied by different authors to obtain a functional form of the deformation curve that must satisfy the boundary conditions, diaphragm geometry, and the specific loading condition. Typical approaches to determine the deformation curve of clamped diaphragms used to design CMUTs can be grouped into 3 main categories: (1) thin plates with small deflection, (2) thin plates with large deflection, and (3) membrane approximation.

(1) Thin Plates with Small Deflection $(w \ll h)$. When the deflection $w$ of a microfabricated thin diaphragm is much small compared to its thickness $h$, the transversal deformation of the diaphragm is dominated by the bending stress and the residual stress developed during the fabrication process. Authors in [5] presented an approximate mathematical expression to determine the deformation curve for thin plates in small deflection regime using a trigonometric series. However, the model is computationally expensive as it requires extensive numerical calculations to determine a set of coefficients. Authors in [6] used the Galerkin method with polynomial basis function to determine the deflection shape. However, the model is limited in accuracy and convergence [7]. Authors in [7] improved the accuracy and convergence of the approach in [6] by replacing the polynomial basis function with a trigonometric basis function. However, the model has not been verified against any finite element analysis (FEA) or experimental results.

(2) Thin Plates with Large Deflection. When the deflection $w$ of a microfabricated thin diaphragm becomes comparable to or larger than its thickness, the strain energy due to stretching of the middle plane of the diaphragm can no longer be ignored. Thus, in case of thin plates with large deflection, approach that accounts for the stretching of the diaphragm midplane along with the bending stress and the residual stress developed during the fabrication process must be used. Functional forms based on polynomials [8] using Ritz energy method and sine/cosine functions in the form of Fourier series using von Karman equations [9] have been explored by many authors. However, these methods are computationally very expensive and an exact solution would require infinite number of terms. In [5], a more compact polynomial-based function that can be solved using energy minimization method has been proposed to determine the deformation curve of thin plates in the large deflection regime. But this too is computationally quite expensive as it involves the determination of eleven undetermined constants by numerical solution of eleven simultaneous nonlinear equations using the method of successive approximation [5].

Authors in [10] proposed a deflection shape function using a single-term Fourier approximation of the exact bending shape that incorporates a double cosine squared term. Due to its simpler form, the function is widely used for load-deflection analysis of clamped diaphragms subject to large deflections. However, its accuracy is compromised due to the truncation of higher order terms in the Fourier series. A similar function based on double sine squared term proposed in [11] also suffers from the same accuracy issue.

(3) Membrane Approximation. The authors in $[5,12]$ have used a membrane approximation to determine the center deflection and the deformation curve for clamped thin diaphragms. For a thin membrane which has much larger lateral dimensions compared to its thickness, strain energy due to bending becomes negligibly small compared to those due to the residual stress and nonlinear stretching of the middle plane. As the bending moment at the clamped edge is zero, unlike the plates, zero gradient of the tangent plane to the displacement surface along the edge is no longer a required boundary condition for membranes [5, 12]. Authors in [5] proposed a membrane deflection shape function based on a double cosine function which is a one term approximation of Fourier series that represents the actual deflection shape. Following the function, the membrane deformation curve along either the $x$ - or $y$-axis mimics the single-mode vibration of a string attached to two ends where the displacements normal to the initial string position at the fixed nodes are zero but the tangents to the string at the nodes are not zero. However, the model fails to describe the diaphragm's real bending shape accurately, and the authors in [12] improved the accuracy of the model by adding two more terms to minimize the deviation from FEA and experimental results. Though the modified model shows excellent agreement with experimental results for thin diaphragms with relatively large sidelengths, investigation by the authors shows that it does not agree well with the deflection shapes of thick diaphragms that behave more like plates. Further, deviation increases monotonically for thinner diaphragms with sidelengths less than $1 \mathrm{~mm}$ to imply that actual behavior of thin diaphragms depends not only on the deflection-thickness ratio, but also on the lateral dimension-thickness ratio.

Typical diaphragms as used in CMUTs have thicknesses in the range of $0.4 \mu \mathrm{m}$ to $1-3 \mu \mathrm{m}$. An analysis carried out by the authors that involves comparison of different models with the FEA and experimental results reveals that even for a very thin diaphragm, the bending moments at the clamped edges cannot be neglected and a membrane approximation would fail to capture the actual deformed shape of the diaphragm. The analysis also reveals that the double cosine or double cosine squared or double sine squared functions also fail to adequately capture the true nature of the deformed shape. The authors observed that the deflection shape functions presented in $[5,10-12]$ deviate considerably in accuracy from 3-D FEA with maximum deviations of $50-60 \%$ for $1-3 \mu \mathrm{m}$ thick and $200-1000 \mu \mathrm{m}$ sidelength diaphragms. Consequently, this deviation ripples into the calculated capacitance values to make the accuracy worse. Investigation by the authors shows that if the existing deflection shape functions are used, resulting capacitance values are in error as high as $16-18 \%$ for a $2 \mu \mathrm{m}$ thick $200 \mu \mathrm{m}$ sidelength square diaphragm CMUT device. 
Moreover, commonly used parallel plate approximation theory for capacitance calculation neglects the fringing field capacitance associated with the diaphragm edges, due to an infinite plate assumption. However, investigation by the authors shows that fringing field capacitance associated with the diaphragm edges also contribute to the total capacitance during deformation.

In this context, this paper develops a new, readily usable, simple, and highly accurate deflection shape function for uniformly loaded clamped square diaphragms used in typical CMUT design space. A polynomial-based function presented in [5] for thin plates in large deflection has been modified to include two empirically determined parameters to develop the new function. The new empirical parameters capture the contributions of the eleven undetermined constants of the polynomial function which can be determined only through extensive numerical analysis [5]. The resulting deflection shape function shows excellent accuracy in the typical CMUT design space and is also applicable to both small and large deflection regimes. Further, a capacitance calculation method has been developed that uses the new deflection shape function and includes fringing field effects to calculate the capacitance of a square diaphragm CMUT with an accuracy higher than those available in the literature.

The rest of the paper has been organized in the following manner: Section 2 gives a brief description of device operation including the problem definition and reviews the deflection shape functions presented in $[5,10,12]$ and their discrepancies with the FEA results; Section 3 describes the development of a new deflection shape function that can more accurately represent the deflection shapes of square diaphragms used in CMUT devices; Section 4 describes capacitance calculation formulae that takes into account the fringing field effect; Sections 5 and 6 provide the validation of the new deflection shape function and the capacitance model by comparing with the FEA results obtained using IntelliSuite and the experimental results published elsewhere. Section 7 concludes the findings of this work.

\section{Problem Illustration}

The structure of a square diaphragm CMUT as shown in Figure 1 consists of a dielectric spacer-supported clamped square diaphragm separated from a fixed backplate by a thin airgap. The diaphragm sidelength is $2 a$, thickness $h$, and the thickness of the airgap is $d_{0}$. When exposed to an external uniform pressure $P_{M}$, the diaphragm deflects causing a decrease in the airgap that results in an increase in capacitance between the diaphragm and the backplate. When pressure is withdrawn, the diaphragm moves back to its original position resulting in a decrease in capacitance. For a time varying incident pressure, the capacitance change follows the same dynamic characteristics of the incident pressure. This change in capacitance is converted into a useful voltage signal using a bias voltage and a charge integrator.

The diaphragm is assumed to be homogeneous and isotropic with perfect edge conditions. It is assumed that

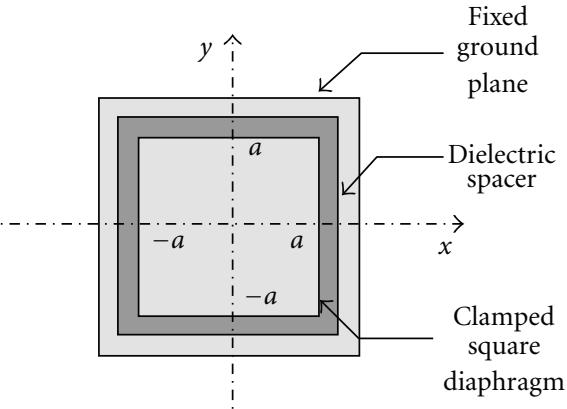

Top view (not to scale)

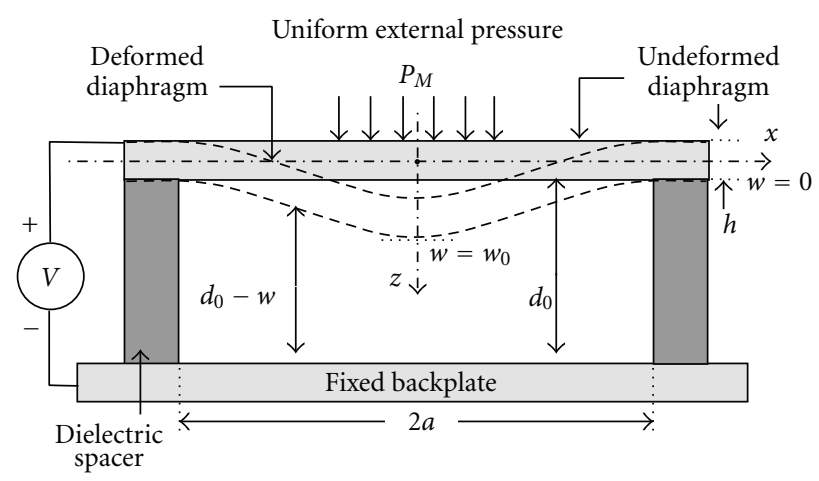

Cross-sectional view

Figure 1: Top and cross-sectional view of a conceptual CMUT device with a square diaphragm.

the clamped edges hold the diaphragm rigidly against any out-of-plane rotation or displacement at the edges but allow displacement parallel to the diaphragm plane. At the edges, out-of-plane displacement is zero and the tangent plane to the displacement surface along the edge coincides with the initial position of the diaphragm middle plane. The boundary conditions imposed by the clamped edges can be expressed mathematically as $[5,10]$

$$
\begin{gathered}
w(x= \pm a, \forall y)=0, \\
w(y= \pm a, \forall x)=0, \\
\frac{d w}{d x}(x= \pm a, \forall y)=0, \\
\frac{d w}{d y}(y= \pm a, \forall x)=0 .
\end{gathered}
$$

Following the variational method, the load-deflection model of a rigidly clamped square diaphragm under large deflection due to an applied uniform pressure $P_{M}$ can be expressed as [13]

$$
P_{M}=\left[C_{r} \frac{\sigma_{0} h}{a^{2}}+C_{b} \frac{12 D}{a^{4}}\right] w_{0}+\left[C_{s} f_{s}(\nu) \frac{\tilde{E} h}{a^{4}}\right] w_{0}^{3},
$$

where $w_{0}$ is the deflection at the center of the diaphragm, $\sigma_{0}$ is residual stress, and $v$ is the Poisson ratio of the diaphragm material. The constants $C_{r}, C_{b}$, and $C_{s}$ are determined by 


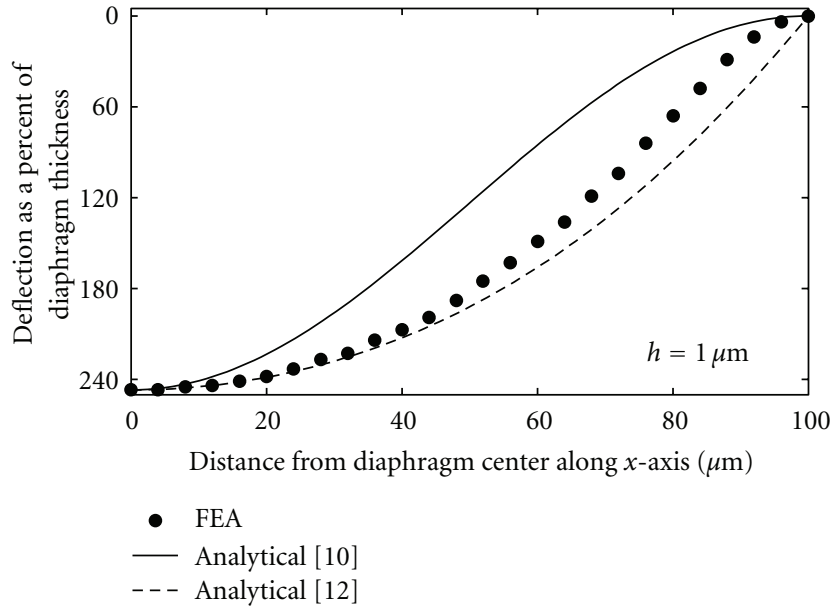

Figure 2: Comparison of FEA deflection profile of $1-\mu \mathrm{m}$ thick polysilicon diaphragm with the existing models, plotted from center to diaphragm edge. $P_{M}=172 \mathrm{kPa}$.

adjusting the analytical solution with the numerical results in $[12]$ as

$$
\begin{gathered}
C_{r}=3.45, \\
C_{b}=4.06, \\
C_{s}=1.994 .
\end{gathered}
$$

The Poisson ratio dependent function $f_{s}(\nu)$ is given by [12]

$$
f_{s}(\nu)=\frac{1-0.271 v}{1-\nu} .
$$

In (2), $D$ is the flexural rigidity of the diaphragm and is expressed as

$$
D=\frac{\tilde{E} h^{3}}{12\left(1-v^{2}\right)}
$$

and the effective Young's modulus $\widetilde{E}$ is the plate modulus, expressed as

$$
\widetilde{E}=\frac{E}{1-v^{2}},
$$

where $E$ is the Young's modulus of the diaphragm material. In (2), the first term within the square bracket on the right side represents diaphragm stiffness due to residual stress, second term is the stiffness due to bending, and the third term represents stiffness due to nonlinear spring hardening. Real root of the above 3rd order polynomial (2) represents the diaphragm center deflection. Two other roots are imaginary and have no practical significance. Once the center deflection is obtained, deformation curve of the diaphragm can be obtained following any of the deflection shape functions discussed in the introduction section.

Figures 2 and 3 show a comparison of FEA determined deflection profiles of two polysilicon diaphragms, 1 and $3 \mu \mathrm{m}$ thick, respectively, with those obtained following the

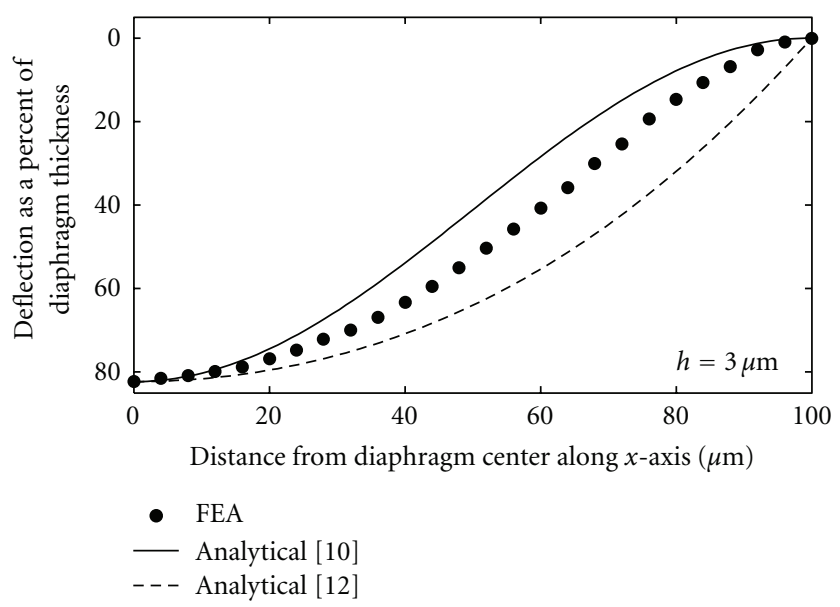

FIgURE 3: Comparison of FEA deflection profile of $3-\mu \mathrm{m}$ thick polysilicon diaphragm with the existing models, plotted from center to diaphragm edge. $P_{M}=950 \mathrm{kPa}$.

TABle 1: Device specifications.

\begin{tabular}{lcc}
\hline Parameter & Value & Unit \\
\hline Diaphragm sidelength & 200 & $\mu \mathrm{m}$ \\
Young's modulus & 169 & $\mathrm{GPa}$ \\
Residual stress & 100 & $\mathrm{MPa}$ \\
Poisson ratio & 0.3 & - \\
\hline
\end{tabular}

deflection shape functions presented in $[10,12]$. These two deflection shape functions are chosen for comparison purposes as they are most widely used, probably due to their simplicity. Device specifications as listed in Table 1 have been used for the FEA simulation. Deflection profiles are plotted from diaphragm center along $x$-axis using the same center deflection value as obtained following FEA for all the cases and are plotted as a percentage of diaphragm thickness. From the figures, it is evident that while [12] agrees closely with the deflection profile of the $1-\mu \mathrm{m}$ thick diaphragm, it predicts significantly higher deflection values compared to FEA for the 3- $\mu \mathrm{m}$ thick diaphragm. In contrast, deflection values calculated following [10] are lower compared to the FEA results for both the 1 and $3 \mu \mathrm{m}$ thick diaphragms with significantly lower deflection values for the $1 \mu \mathrm{m}$ thick diaphragm. Also, the FEA results show relatively larger drooping in the deflection profiles for the thinner diaphragm than the thicker one. This behavior is expected as the thinner diaphragms are stress dominated, whereas the thicker ones behave more like plates (bending dominated).

Figures 4 and 5 show the deflection profiles for the same diaphragms but plotted from the center along a diagonal direction towards the top right corner of the diaphragm. From the figures, the maximum percent deviations of the profiles following $[10,12]$ relative to the FEA results are estimated to be around $57 \%$ and $68 \%$, respectively. These deviations are too large to ignore in any design process. It is obvious from the results that while [10] is more accurate for the deflection profile of thick diaphragms, [12] is more accurate for the thinner ones. However, both of 


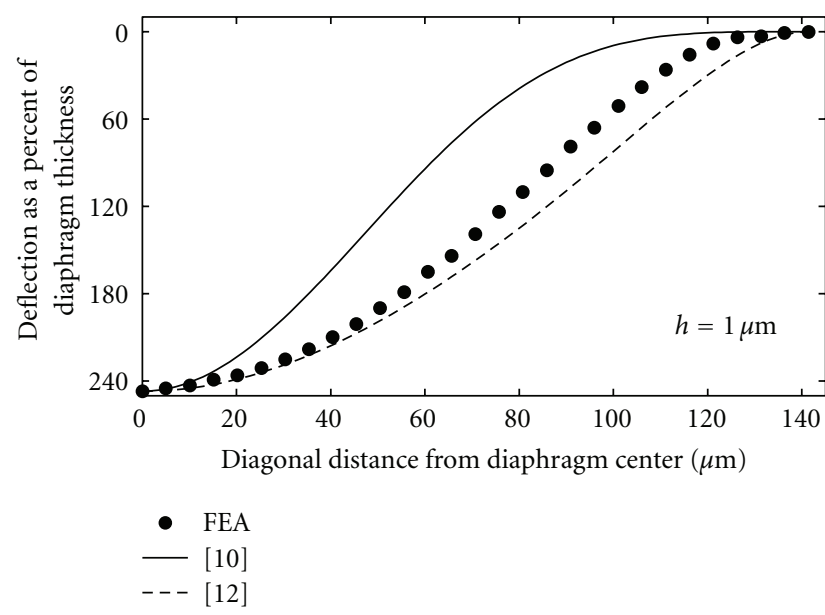

Figure 4: Comparison of FEA deflection profile of $1-\mu \mathrm{m}$ thick polysilicon diaphragm with the existing models, plotted from center along the diagonal axis of the diaphragm. $P_{M}=172 \mathrm{kPa}$.

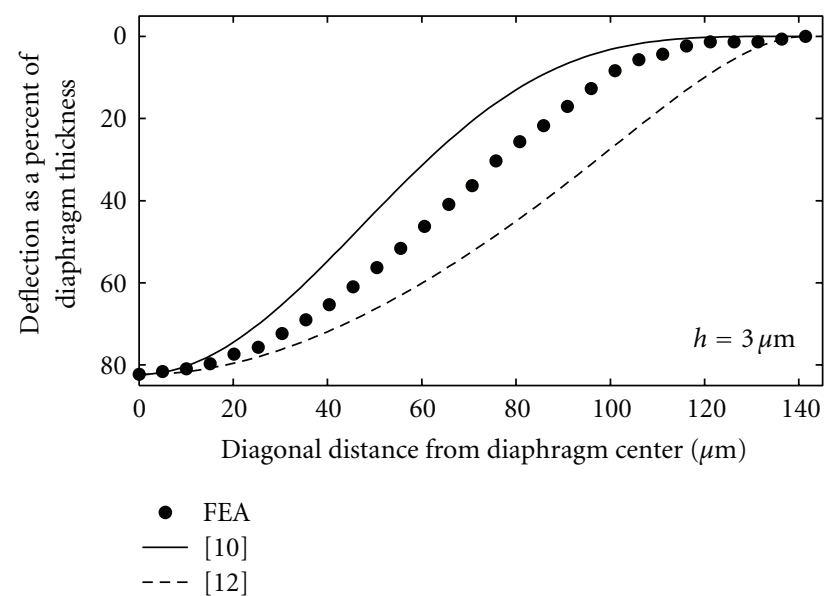

Figure 5: Comparison of FEA deflection profile of $3-\mu \mathrm{m}$ thick polysilicon diaphragm with the existing models, plotted from center along the diagonal axis of the diaphragm. $P_{M}=950 \mathrm{kPa}$.

them deviate significantly from the 3-D FEA results for diaphragms typically used in CMUT design space.

As the diaphragm lies in the $x-y$ plane, the parallel plate capacitance between the deformed diaphragm and the backplate can be calculated following [14]

$$
C_{\text {Deform }}=\varepsilon_{0} \iint_{A} \frac{d x d y}{d_{0}-w(x, y)},
$$

where $\varepsilon_{0}$ is the permittivity of free space and $w(x, y)$ represents the deflection surface of deformed diaphragm also known as deflection shape function.

From the Figures $2-5$, it is evident that the existing deflection shape functions are not adequate to accurately describe the deflection profiles of clamped square diaphragms within the design space considered in this work. Thus, use of them, notably $[10,12]$, would result in significant error if used in (7) to calculate the capacitance.

\section{New Deflection Shape Function}

The new deflection shape function has been developed by modifying the equations presented in [5] for thin plates in large deflection. Following [5], the lateral and transverse displacements of a rigidly clamped thin plate with sidelengths $2 a$ and $2 b$ can be approximated by

$$
\begin{gathered}
u=\left(a^{2}-x^{2}\right)\left(b^{2}-y^{2}\right) x\left(b_{00}+b_{02} y^{2}+b_{20} x^{2}+b_{22} x^{2} y^{2}\right), \\
v=\left(a^{2}-x^{2}\right)\left(b^{2}-y^{2}\right) y\left(c_{00}+c_{02} y^{2}+c_{20} x^{2}+c_{22} x^{2} y^{2}\right), \\
w=\left(a^{2}-x^{2}\right)^{2}\left(b^{2}-y^{2}\right)^{2}\left(a_{00}+a_{02} y^{2}+a_{20} x^{2}\right),
\end{gathered}
$$

where $u$ and $v$ are the displacements in the middle plane of the plate along $x$ and $y$ directions, respectively, and vanish at the boundary and $w$ represents the transverse deflection. As $w$ also vanishes at the boundary and so does its first derivative, all the necessary boundary conditions imposed by the clamped edges are satisfied by (8). The undetermined parameters $b_{00}, \ldots, a_{20}$ in (8) can be determined by applying the principle of virtual displacement and an energy minimization method [5]. However, determination of eleven undetermined parameters is computationally quite expensive as it will involve numerical solutions of eleven simultaneous nonlinear equations using the method of successive approximation. Most notable feature of $(8)$ is that the polynomial basis function $\left(a^{2}-x^{2}\right)^{2}\left(b^{2}-y^{2}\right)^{2}$ captures the actual shape of the deformation curve with an accuracy higher than the double cosine squared [10] or double sine squared [11] functions and the quantity $a_{00}$ is associated with the center deflection of the diaphragm that can be calculated following (2). Instead of trying to determine eleven undetermined parameters by solving eleven simultaneous nonlinear equations using the method of successive approximation each time, a simple and straightforward approach can be to formulate a deflection shape function with the same basis function as in (8) and then determine one or two empirical parameters that capture the contribution of eleven unknown parameter values with reasonable accuracy for a target design space. Following this approach, a trial deflection shape function

$$
w=w_{0}\left(1-\frac{x^{2}}{a^{2}}\right)^{2}\left(1-\frac{y^{2}}{a^{2}}\right)^{2}\left[1+c_{1}\left(\frac{x^{2}+y^{2}}{a^{2}}\right)\right]
$$

has been formulated where the second term in the square bracket represents the adjustment necessary to compensate for the deviation of the polynomial function $w_{0}\left(1-x^{2} / a^{2}\right)^{2}\left(1-y^{2} / a^{2}\right)^{2}$ from the actual deflection shape. The coefficient $c_{1}$ can be determined for any target design space by comparing with the deflection profiles obtained experimentally or from FEA analysis. To investigate the validity of the trial function (9), deflection profiles obtained following (9) for $c_{1}=0$ (dashed line) and $c_{1}=1.1$ (solid line) are compared in Figure 6 with the FEA-determined deflection profile of a polysilicon diaphragm for small diaphragm deflection ( $w_{0}$ about $21 \%$ of the diaphragm thickness). As can be seen from the figure, deflection profile 


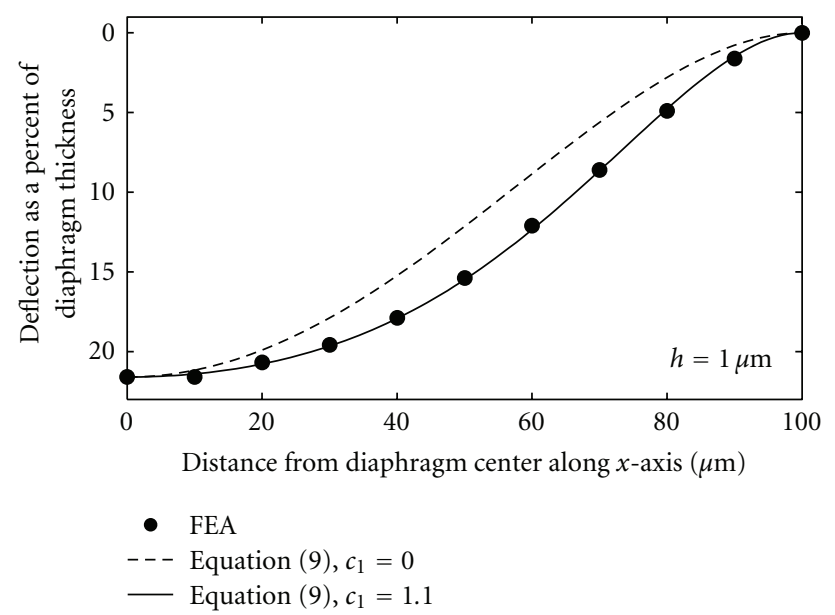

FIgURE 6: Comparison of FEA deflection profile of $1-\mu \mathrm{m}$ thick polysilicon diaphragm with the trial deflection shape function (9) for small deflection, plotted from center to diaphragm edge. $P_{M}=$ $10 \mathrm{kPa}$.

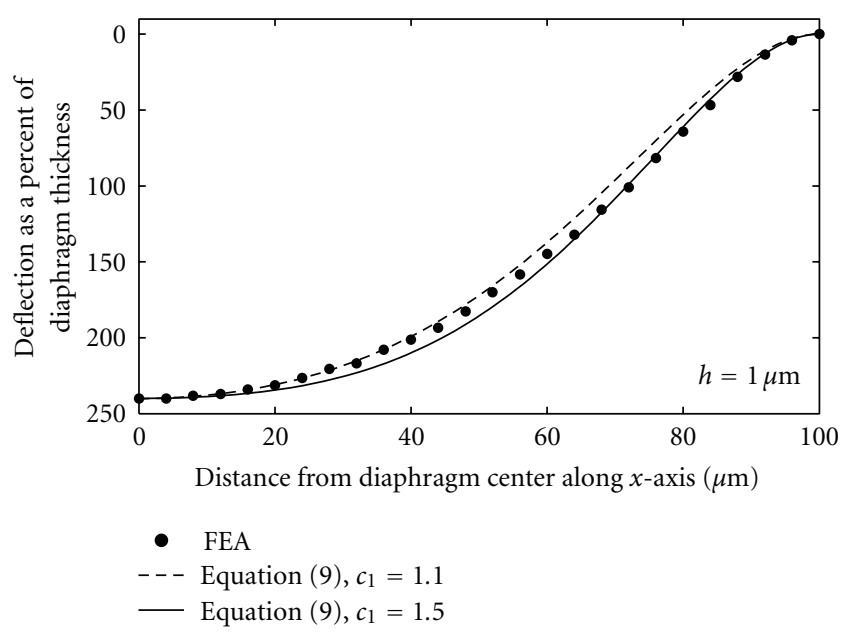

FIgURE 7: Comparison of FEA deflection profile of $1-\mu \mathrm{m}$ thick polysilicon diaphragm with the trial deflection shape function (9) for large deflection, plotted from center to diaphragm edge, with $c_{1}=1.1$ (dashed line) and $c_{1}=1.5$ (solid line). $P_{M}=172 \mathrm{kPa}$.

obtained following the trial function with $c_{1}=1.1$ shows excellent agreement with the FEA derive deflection profile for small diaphragm deflection.

To investigate the effectiveness of (9) for diaphragms subject to large deflection, a similar comparative plot of deflection profiles of the polysilicon diaphragm subjected to a uniform pressure of $172 \mathrm{kPa}$, obtained following FEA and the trial function (9) for $c_{1}=1.1$ (dashed line) and $c_{1}=1.5$ (solid line), is plotted in Figure 7. The maximum diaphragm deflection $w_{0}$ is set at $240 \%$ of the diaphragm thickness. It can be observed from Figure 7 that while for $c_{1}=1.1$, (9) predicted deflection profile agrees well with the FEA results for $0<x<0.5 a$, for $c_{1}=1.5$, it agrees well with FEA results for $0.65 a<x<a$. These observations suggest that though the trial function (9) provides accuracy better than those that can

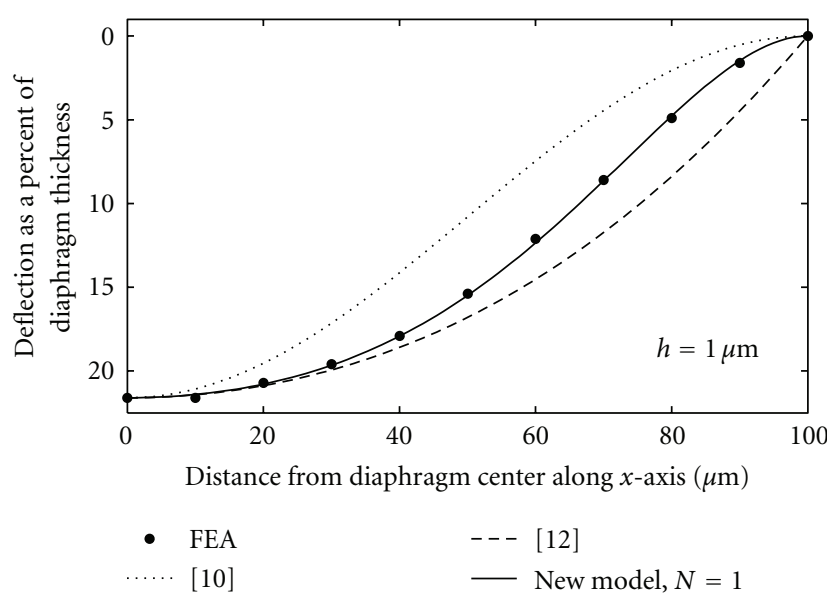

FIGURE 8: Comparison of FEA deflection profile with the new and the existing models for the $1-\mu \mathrm{m}$ thick polysilicon diaphragm for small deflection, plotted from center to diaphragm edge. $P_{M}=$ $10 \mathrm{kPa}$.

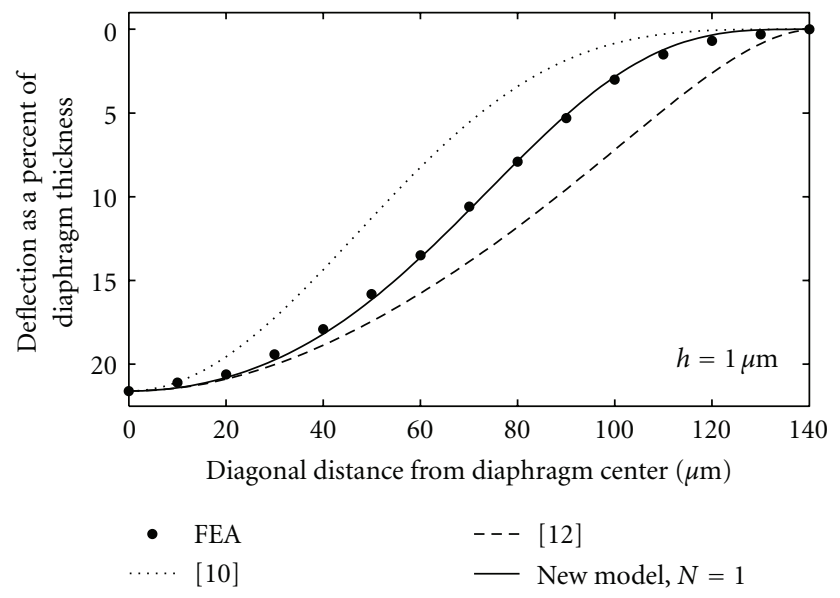

FIGURE 9: Comparison of FEA deflection profile with the new and the existing models for the $1-\mu \mathrm{m}$ thick polysilicon diaphragm for small deflection, plotted along the diagonal axis of the diaphragm. $P_{M}=10 \mathrm{kPa}$.

be achieved by either [10] or [12], the accuracy can be further improved by adjusting $c_{1}$ and introducing additional terms in (9) to adequately capture the nonlinearity of the diaphragm behavior during large deflection. Therefore, a strategy has been adopted to determine optimal value of $c_{1}$ to match the deflection profile obtained following (9) to some part of the FEA result and then introduce one or more terms in (9) to compensate for the deviation of (9) from the rest of the FEA simulation result.

By careful investigation, it has been determined that a fourth order term in the form of $\left(x^{4} / a^{4}\right)$ can minimize the deviation in the $x>0.5 a$ range. The rationale behind this is that because of the higher order, the term would have negligible effect on deflection for smaller values of $x$ $(0<x<0.5 a)$. Due to symmetry, same argument applies to diaphragm deflection along $y$ direction. Following this 
methodology, a new term $c_{2}\left(x^{2}+y^{2}\right)^{2} / a^{4}$ is introduced in (9) to obtain the new deflection shape function as

$$
\begin{array}{r}
w=w_{0}\left(1-\frac{x^{2}}{a^{2}}\right)^{2}\left(1-\frac{y^{2}}{a^{2}}\right)^{2}[ \\
{\left[+c_{1}\left(\frac{x^{2}+y^{2}}{a^{2}}\right)\right.} \\
\left.+c_{2}\left(\frac{x^{2}+y^{2}}{a^{2}}\right)^{2}\right] .
\end{array}
$$

The above equation can be rewritten in its general form as

$$
w=w_{0}\left(1-\frac{x^{2}}{a^{2}}\right)^{2}\left(1-\frac{y^{2}}{a^{2}}\right)^{2} \sum_{n=0,1,2}^{N} c_{n}\left(\frac{x^{2}+y^{2}}{a^{2}}\right)^{n},
$$

where the coefficients $c_{n}$ are adjustable for any design space. For the target CMUT design space (diaphragm thickness range of $1-3 \mu \mathrm{m}$ and sidelength range of $200-1000 \mu \mathrm{m}$ ), investigation shows that three terms $(N=2)$ in (11) are necessary for large deflection cases while only two terms $(N=1)$ are necessary for small deflection cases to achieve a high degree of accuracy. For the specified design space, the parameters $c_{0}, c_{1}$, and $c_{2}$ have been determined as

$$
\begin{gathered}
c_{0}=1, \\
c_{1}=\frac{0.0011}{\sqrt{h}}, \\
c_{2}=\frac{0.0005}{\sqrt{h}},
\end{gathered}
$$

by comparing the results from (11) with 3-D FEA using IntelliSuite for a wide range of device specifications and loading conditions.

\section{Capacitance Calculation}

Commonly used parallel plate capacitance model as expressed in (7) does not take account of the fringing field effects. However, investigation by the authors show that for diaphragms with smaller sidelength $(2 a<1 \mathrm{~mm})$, contribution of fringing field capacitance goes up and can be as high as $9 \%$ for diaphragms with sidelength $100 \mu \mathrm{m}$ and airgap $3 \mu \mathrm{m}$. Therefore, it is necessary to include the fringing field capacitance while calculating the total capacitance of CMUTs which are typically built with diaphragms with sidelengths less than $1 \mathrm{~mm}$.

Though an accurate value of the fringing field capacitance can only be obtained by solving Poisson's equation using a 3D field solver, an approximate value of the fringing field capacitance can be obtained by modifying a formula presented in [15] to calculate the capacitance between a VLSI on-chip interconnect separated from an underneath silicon substrate by a dielectric medium of thickness $d_{0}$, expressed as

$$
C=\varepsilon L\left[\frac{W}{d_{0}}+0.77+1.06\left(\frac{W}{d_{0}}\right)^{0.25}+1.06\left(\frac{h}{d_{0}}\right)^{0.5}\right],
$$

where $L$ is the length and $W$ is the width of the metal interconnect, and $\varepsilon=\varepsilon_{0} \varepsilon_{r}$, where $\varepsilon_{r}$ is relative permittivity of the dielectric layer. The quantity $\varepsilon L W / d_{0}$ in (13) is simply the parallel plate capacitance. The second term within the square bracket represents fringing field capacitance due to the interconnect length $(L)$, the third term due to interconnect width $(W)$, and the fourth term represents that due to the interconnect thickness $(h)$. Rearranging (13), fringing field capacitances can be expressed as a function of parallel plate capacitance in the form:

$$
C=C_{0}\left(1+C_{\mathrm{ff}}\right),
$$

where $C_{0}$ is the parallel plate capacitance $\left(\varepsilon L W / d_{0}\right)$ and $C_{\mathrm{ff}}$ is the fringing field factor expressed as

$$
C_{\mathrm{ff}}=0.77 \frac{d_{o}}{W}+1.06\left(\frac{d_{o}}{W}\right)^{0.75}+1.06 \frac{\left(h d_{0}\right)^{0.5}}{W} .
$$

Investigation shows that (15) can be used to calculate the capacitance of a rigidly clamped square diaphragm separated from the ground plane by a small airgap $\left(\varepsilon_{r}=1\right)$. The third term in (15) representing the fringing field capacitance due to diaphragm thickness can be neglected as the flux lines originating from the diaphragm sides along the thickness will terminate beyond the effective area of the backplate of the device and, therefore, will not contribute to the total capacitance. Thus, for a square diaphragm with sidelength $W=L=2 a$, the capacitance between the undeflected diaphragm and the backplate can be expressed as

$$
C=C_{0}\left(1+C_{\mathrm{ff}}\right)=\frac{\varepsilon_{0} 4 a^{2}}{d_{0}}\left[1+0.77\left(\frac{d_{0}}{2 a}\right)+1.06\left(\frac{d_{0}}{2 a}\right)^{0.75}\right] .
$$

After deformation, the total capacitance is also contributed by two factors: the parallel plate capacitance $C_{\text {Deform }}$ between the deformed diaphragm and the backplate which can be calculated using (7) and the fringing field capacitance $C_{\text {Deform }} C_{\text {ff. }}$. Thus, the total capacitance after deformation can be expressed as

$$
C=C_{\text {Deform }}\left(1+C_{\mathrm{ff}}\right) .
$$

Since diaphragm edges are rigidly fixed and do not undergo any deformation and as fringing field is contributed mainly by the charges concentrated at the edges, fringing field factor $C_{\mathrm{ff}}$ can be assumed to remain unchanged despite diaphragm deformation and the same formula (15) can be used to calculate $C_{\mathrm{ff}}$ as before.

\section{FEA Validation}

\subsection{Deflection Shape Function}

(i) Thin Plates in Small Deflection. Figure 8 shows comparison of the FEA-derived-deflection profile of a $1 \mu \mathrm{m}$ thick polysilicon diaphragm for small diaphragm deflection $\left(w_{0} \cong\right.$ $21 \%$ of the diaphragm thickness), plotted from center to the midpoint of the edge along the $x$-axis, with those obtained following the new (11) and the existing models $[10,12]$. The diaphragm specifications are as listed in Table 1 . The figure 
TABLE 2: Comparison of new model-calculated capacitance values using deflection shape functions following [10, 12] and (11) with the FEA results for small diaphragm deflection. $d_{0}=3 \mu \mathrm{m}$, Young's Modulus, $E=169 \mathrm{GPa}$, Poisson Ratio, $v=0.3$, and Bias Voltage $=12 \mathrm{~V}$ for all cases.

\begin{tabular}{|c|c|c|c|c|c|c|c|c|c|c|c|}
\hline \multirow{2}{*}{$a$} & \multirow{2}{*}{$h$} & \multirow{2}{*}{$\sigma_{0}$} & \multirow{2}{*}{$P_{M}$} & \multirow{2}{*}{$w_{0}$} & \multirow{2}{*}{ C } & \multicolumn{3}{|c|}{$C$ analytical (fF) } & \multicolumn{3}{|c|}{$\% \Delta C$ (FEA-analytical) } \\
\hline & & & & & & {$[10]$} & {$[12]$} & New model & {$[10]$} & {$[12]$} & New model \\
\hline$(\mu \mathrm{m})$ & $(\mu \mathrm{m})$ & $\mathrm{MPa}$ & $(\mathrm{kPa})$ & $\begin{array}{l}\text { FEA } \\
(\mu \mathrm{m})\end{array}$ & FEA (fF) & $N=1$ & $N=1$ & & & & \\
\hline 100 & 1 & 100 & 20 & 0.43 & 132.96 & 130.01 & 135.06 & 132.72 & 2.22 & -1.58 & 0.18 \\
\hline 100 & 2 & 100 & 160 & 1.08 & 144.84 & 139.22 & 154.01 & 145.5 & 3.88 & -6.33 & -0.46 \\
\hline 100 & 3 & 100 & 400 & 1.18 & 146.08 & 140.3 & 156.4 & 146.3 & 3.94 & -7.05 & -0.15 \\
\hline 100 & 1 & 250 & 60 & 0.43 & 136.64 & 132.14 & 139.36 & 136.03 & 3.29 & -1.99 & 0.44 \\
\hline 100 & 2 & 250 & 320 & 0.95 & 149.64 & 142.25 & 160.49 & 150.02 & 4.94 & -7.25 & -0.25 \\
\hline 100 & 3 & 250 & 700 & 1.11 & 151.84 & 144.48 & 165.31 & 152.24 & 4.85 & -8.87 & -0.26 \\
\hline
\end{tabular}

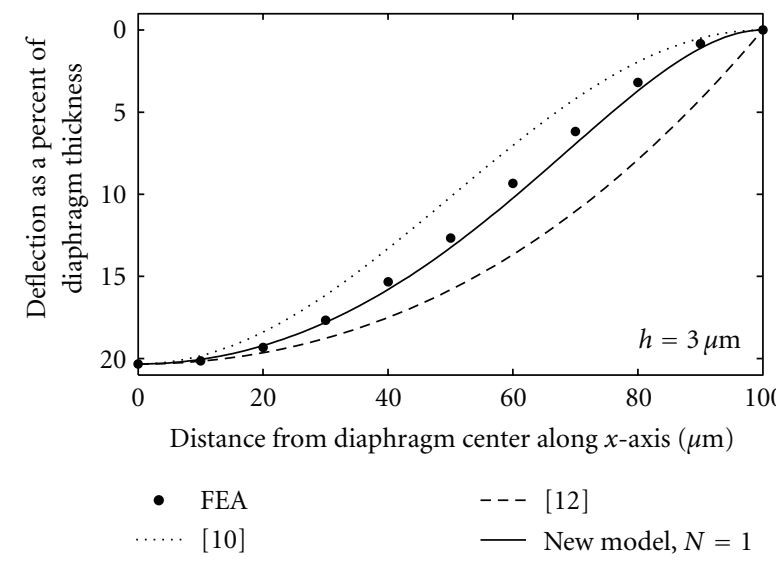

FIGURE 10: Comparison of FEA deflection profile with the new and the existing models for the $3-\mu \mathrm{m}$ thick polysilicon diaphragm for small deflection, plotted from center to diaphragm edge. $P_{M}=$ $200 \mathrm{kPa}$.

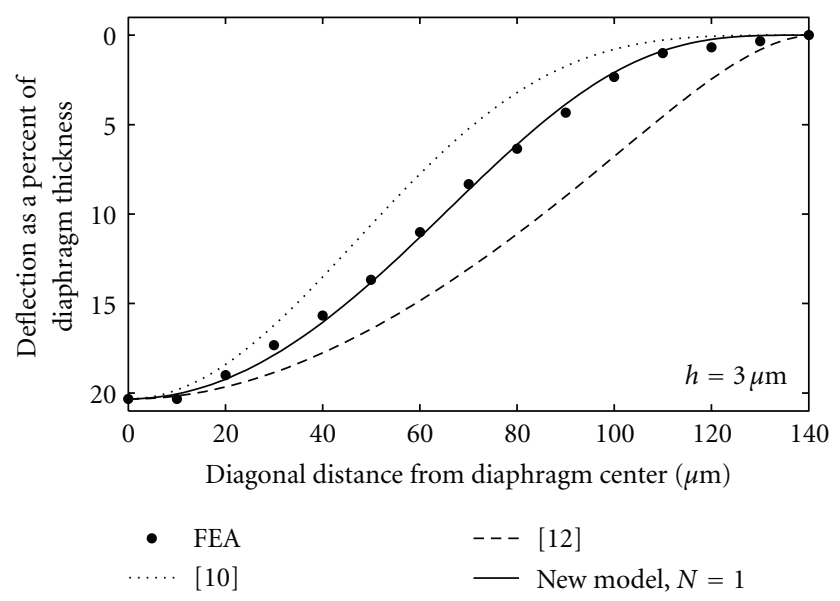

FIGURE 11: Comparison of FEA deflection profile with the new and the existing models for the $3-\mu \mathrm{m}$ thick polysilicon diaphragm for small deflection, plotted along the diagonal axis of the diaphragm. $P_{M}=200 \mathrm{kPa}$. shows excellent agreement between the FEA and the new model predicted deflection profile obtained with $N=1$ in (11). Figure 9 shows excellent agreement between the new model and FEA results for the deflection profile of the same diaphragm along a diagonal line from center to the top right corner of the diaphragm.

Similar comparisons for a $3 \mu \mathrm{m}$ thick polysilicon diaphragm subjected to an external pressure of $200 \mathrm{kPa}$ are shown in Figure 10 (drawn from center along the $x$-axis) and Figure 11 (diagonally from center to the top right corner). Other specifications for the $3 \mu \mathrm{m}$ thick diaphragm remain the same as in Table 1. Figures 8-11 clearly establish that the deflection profiles obtained following the new deflection shape function are in excellent agreement with the FEA results for small deflections.

(ii) Thin Plates in Large Deflection. For large deflections, best match to the FEA results with the new model is obtained with $N=2$ in (11). Figures 12 and 13 show comparison of FEA-derived deflection profiles of a $1 \mu \mathrm{m}$ thick polysilicon diaphragm with the new and the existing models for large diaphragm deflection $\left(w_{0} \cong 240 \%\right.$ of the diaphragm thickness), drawn from center along the $x$-axis and diagonally from center to the top right corner, respectively. Similar comparisons for the $3 \mu \mathrm{m}$ thick diaphragm for large deflection are shown in Figure 14 (drawn from center along the $x$-axis) and Figure 15 (diagonally from center to the top right corner). All the figures show excellent agreement between the new deflection shape function and FEA results for both the thin and thick diaphragms in large deflection.

Figure 16 shows a comparison of the FEA and the new model predicted deflection profiles for the $1-\mu \mathrm{m}$ thick polysilicon diaphragm at different loading conditions resulting in a center deflection as high as 8 times the diaphragm thickness. For all the cases, device specifications as listed in Table 1 are used. The results are still in excellent agreement except for very large deflection (8 times the diaphragm thickness) where (11) predicted deflection profile deviates slightly from the FEA results for $x>0.5 a$.

Figure 17 shows a comparison of the FEA and the new model-predicted deflection profiles for the $1-\mu \mathrm{m}$ thick polysilicon diaphragm with different sidelengths subject 


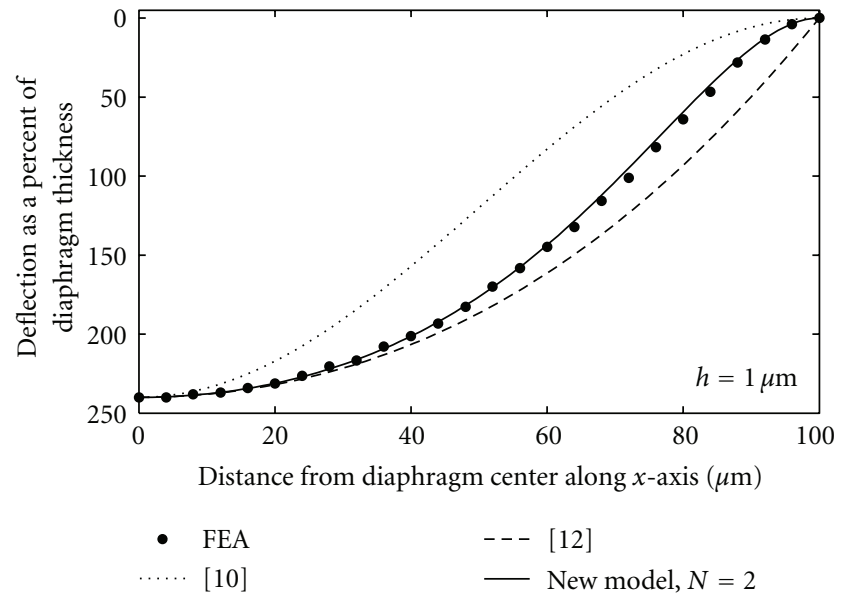

Figure 12: Comparison of FEA deflection profile with the new and the existing models for the $1-\mu \mathrm{m}$ thick polysilicon diaphragm for large deflection, plotted from center to diaphragm edge. $P_{M}=$ $172 \mathrm{kPa}$.

to different external loading conditions. Again excellent agreement between the FEA and the new model is observed except for the diaphragm with large sidelength $(2 a=$ $1000 \mu \mathrm{m}$ ) where the new model-predicted deflection profile deviates slightly from FEA for $x>0.7 a$. The figures clearly establish that the developed deflection shape function (11) can predict deflection profiles of clamped square diaphragms subject to uniform pressure loading with a much higher accuracy compared to the existing models presented in [10] or [12].

5.2. Capacitance Comparison. Capacitance values between the deformed diaphragm and a fixed plate separated by an airgap thickness $d_{0}=3 \mu \mathrm{m}$ have been calculated using (17) for different device parameters and material properties under different external pressure. For the purpose of comparison, deflection profile $w(x, y)$ is calculated using both the existing deflection shape functions following $[10,12]$ and the new model (11). The diaphragm center deflection $w_{0}$ is determined by solving (2). The capacitance values thus calculated using the three deflection shape functions along with those obtained from 3-D FEA under the same conditions are tabulated in Tables 2 and 3 for the small $\left(w_{0} \leq\right.$ $0.5 h)$ and large $\left(w_{0}>0.5 h\right)$ deflections, respectively. While the capacitance values obtained following the new model are in excellent agreement with the FEA results for all the cases with a maximum deviation of $\sim 1.5 \%$, those obtained following $[10,12]$ show large deviations. Deflection shape function in [10] results in an underestimation of capacitance values with a maximum deviation of $\sim 16 \%$, while [12] results in an overestimation with a maximum deviation of $\sim 19 \%$. It is worth mentioning here the effect of fringing fields which when ignored results in a maximum deviation of $\sim 6 \%$ in capacitance values even with the new deflection shape function.

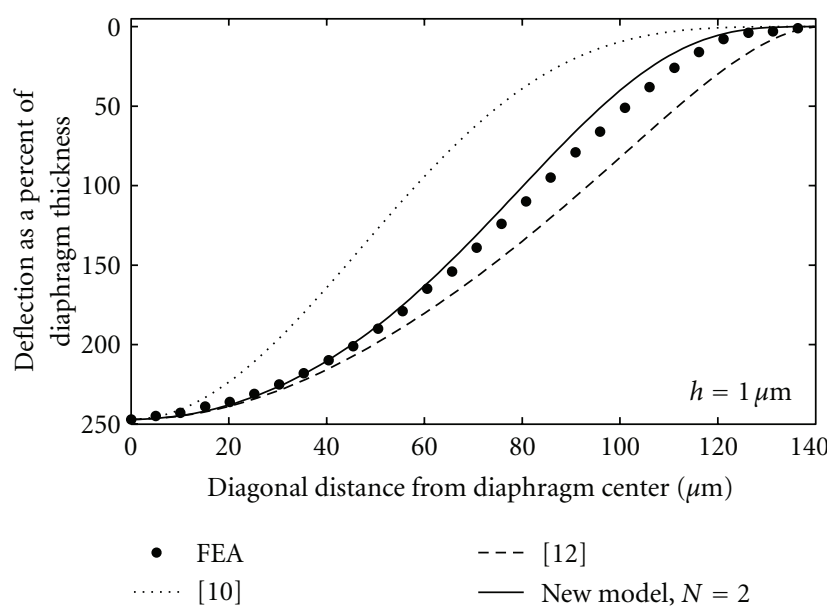

FIGURE 13: Comparison of FEA deflection profile with the new and the existing models for the $1-\mu \mathrm{m}$ thick polysilicon diaphragm for large deflection, plotted from center along the diagonal axis of the diaphragm. $P_{M}=172 \mathrm{kPa}$.

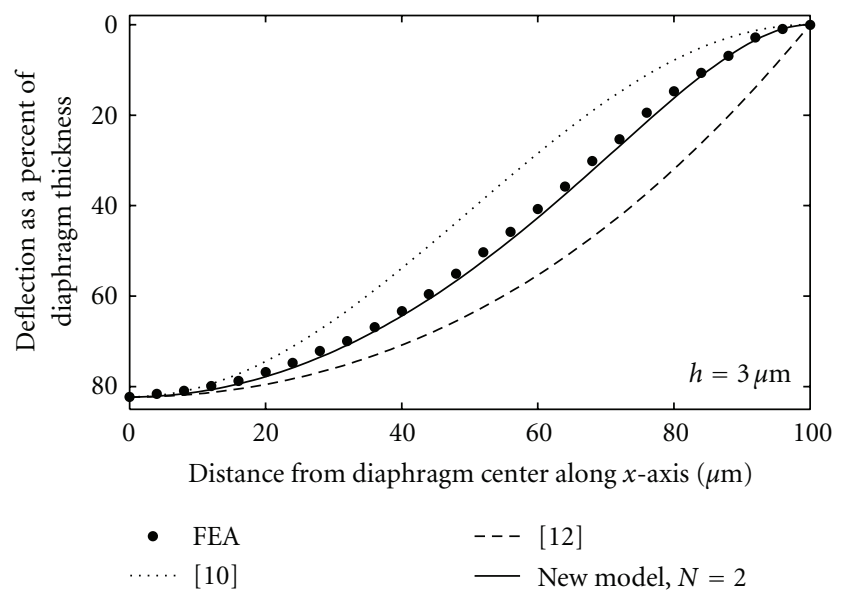

Figure 14: Comparison of FEA deflection profile with the new and the existing models for the $3-\mu \mathrm{m}$ thick polysilicon diaphragm for large deflection, plotted from center to diaphragm edge. $P_{M}=$ $950 \mathrm{kPa}$.

The results clearly indicate that the new model for capacitance calculation when combined with the new deflection shape function predicts the capacitance values of CMUT devices with square diaphragm with a very high degree of accuracy. It is to be mentioned here that 3-D electromechanical analysis module of IntelliSuite uses FASTCAP to calculate the capacitance and the accuracy of FASTCAP is within 1\% of actual values [16].

\section{Experimental Verification}

This section provides experimental verification of the new deflection shape function and the developed model for capacitance calculation. The new deflection shape function is verified against the experimental deflection profile presented 
TABLE 3: Comparison of new model-calculated capacitance values using deflection shape functions following [10, 12] and (11) with the FEA results for large diaphragm deflection. Other parameters same as in Table 2.

\begin{tabular}{|c|c|c|c|c|c|c|c|c|c|c|c|}
\hline \multirow{2}{*}{$a$} & \multirow{2}{*}{$h$} & \multirow{2}{*}{$\sigma_{0}$} & \multirow{2}{*}{$P_{\mathrm{M}}$} & \multirow{2}{*}{$w_{0}$} & \multirow{2}{*}{$C$} & \multicolumn{3}{|c|}{$C$ analytical (fF) } & \multicolumn{3}{|c|}{$\% \Delta C($ FEA-analytical $)$} \\
\hline & & & & & & {$[10]$} & {$[12]$} & New model & {$[10]$} & {$[12]$} & New model \\
\hline$(\mu \mathrm{m})$ & $(\mu \mathrm{m})$ & $\mathrm{MPa}$ & $(\mathrm{kPa})$ & $\begin{array}{l}\text { FEA } \\
(\mu \mathrm{m})\end{array}$ & FEA (fF) & $N=2$ & $N=2$ & & & & \\
\hline 100 & 1 & 100 & 60 & 1.16 & 149.8 & 141.26 & 158.36 & 152.07 & 5.70 & -5.72 & -1.51 \\
\hline 100 & 2 & 100 & 480 & 2.50 & 217.08 & 181.4 & 256.45 & 218.36 & 16.43 & -18.14 & -0.59 \\
\hline 100 & 3 & 100 & 1000 & 2.49 & 209.2 & 178.77 & 249.12 & 208.18 & 14.55 & -19.08 & 0.49 \\
\hline 100 & 1 & 250 & 120 & 1.12 & 150.24 & 140.88 & 157.55 & 151.41 & 6.23 & -4.86 & -0.78 \\
\hline 100 & 2 & 250 & 320 & 1.20 & 149.64 & 142.25 & 160.50 & 151.03 & 4.93 & -7.35 & -0.93 \\
\hline 100 & 3 & 250 & 1000 & 1.85 & 169.6 & 156.72 & 193.08 & 171.89 & 7.59 & -13.84 & -1.35 \\
\hline
\end{tabular}

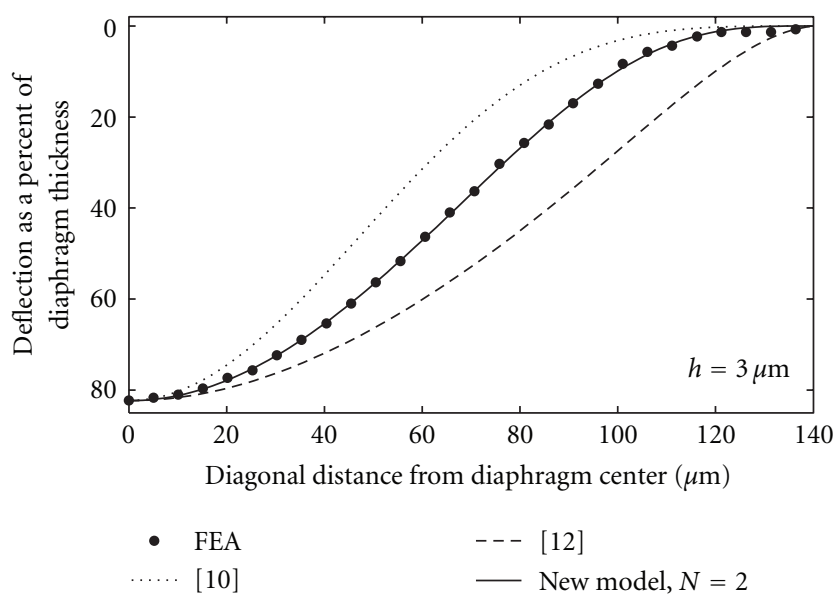

Figure 15: Comparison of FEA deflection profile with the new and the existing models for the $3-\mu \mathrm{m}$ thick polysilicon diaphragm for large deflection, plotted along the diagonal axis of the diaphragm. $P_{M}=950 \mathrm{kPa}$.

in [17] and the developed model for capacitance calculation against the experimental capacitance values of a single-chip MEMS capacitive microphone, measured at different bias voltages from $0-100$ volt, presented in [18].

6.1. Deflection Shape Function. Figure 18 shows comparison of the new model-predicted deflection profile with the experimentally obtained deflection profile of a $13 \mu \mathrm{m}$ thick singlecrystal silicon diaphragm, size $1000 \times 1000 \mu \mathrm{m}^{2}$, subject to a uniform external pressure of $100 \mathrm{kPa}$, presented in [17]. For comparison purpose, deflection profiles following $[10,12]$ are also included in the figure. Analytical profiles are drawn using the same center deflection $w_{0}$ as obtained experimentally. Deflection profiles following the new model are plotted with both $N=1$ and $N=2$, and they almost overlap each other. Due to the large diaphragm thickness $(13 \mu \mathrm{m})$, the third term in (11) has only marginal contribution to the overall diaphragm deflection. As can be seen from the figure, the new model-predicted deflection profiles closely follow the experimental profile for the whole range. However, deflection profiles following $[10,12]$ deviate

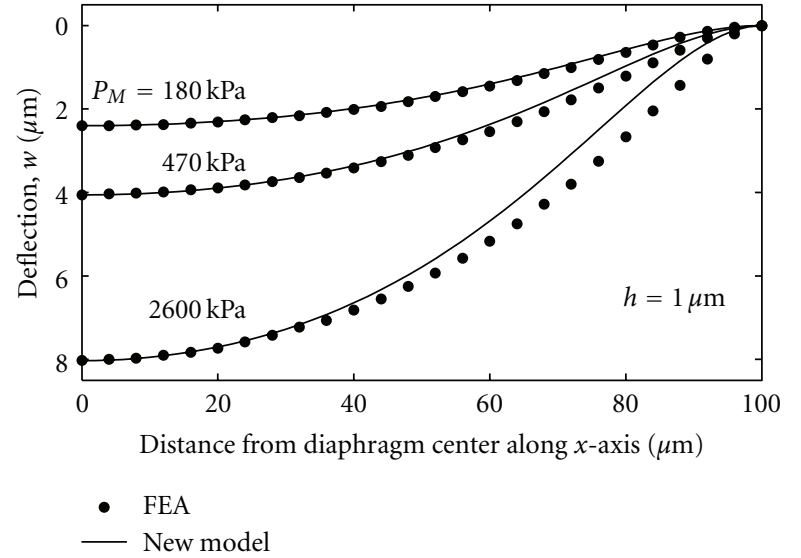

Figure 16: Comparison of FEA deflection profiles with the new model for the 1- $\mu \mathrm{m}$ thick polysilicon diaphragm under different external pressure $P_{M}$, plotted from center to diaphragm edge.

from the experimental profile by a large margin with maximum deviation occurring at around $x=300 \mu \mathrm{m}$. The estimated deviation relative to the experimental value at $x=$ $300 \mu \mathrm{m}$ for the three deflection shape functions following $[10,12]$, and this model are around $42 \%, 27 \%$ and $4 \%$, respectively.

6.2. Capacitance Comparison. In order to verify the accuracy of the developed model for capacitance calculation, we consider the single-chip MEMS capacitive microphone presented in [18]. It consists of a $3 \mu \mathrm{m}$ thick Al diaphragm, size $0.5 \mathrm{~mm} \times 0.5 \mathrm{~mm}$, with acoustic holes separated from a rigid backplate electrode with an air gap of about $1 \mu \mathrm{m}$. The density of the $20 \mu \mathrm{m} \times 20 \mu \mathrm{m}$ acoustic holes is $144 / \mathrm{mm}^{2}$. The Young's modulus of Al is $64 \mathrm{GPa}$, Poisson's ratio 0.36 , and the residual stress is estimated to be around $1500 \mathrm{MPa}$. Figure 19 shows the plots of experimentally determined capacitance values against bias voltage of the said microphone in [18] together with those obtained analytically following (17) using different deflection shape functions.

For calculation of deflection profiles, experimentally determined center deflection values at different bias voltages as obtained from [18] were used. Due to the presence of 


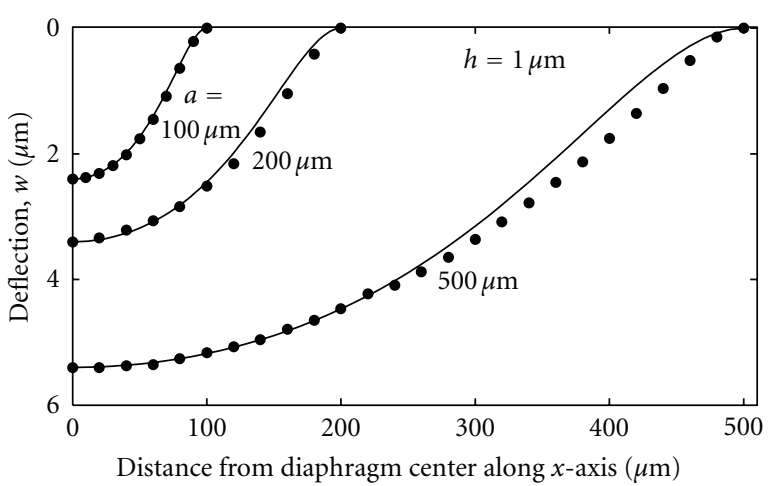

- $\quad$ FEA

- New model

Figure 17: Comparison of FEA deflection profiles with the new model for the $1-\mu \mathrm{m}$ thick polysilicon diaphragms with different sidelengths, plotted from center to diaphragm edge. $\sigma_{0}=$ $100 \mathrm{MPa}, E=169 \mathrm{GPa}, v=0.3, P_{M}=172,44$, and $8.9 \mathrm{kPa}$ for $a=100,200$, and $500 \mu \mathrm{m}$, respectively.

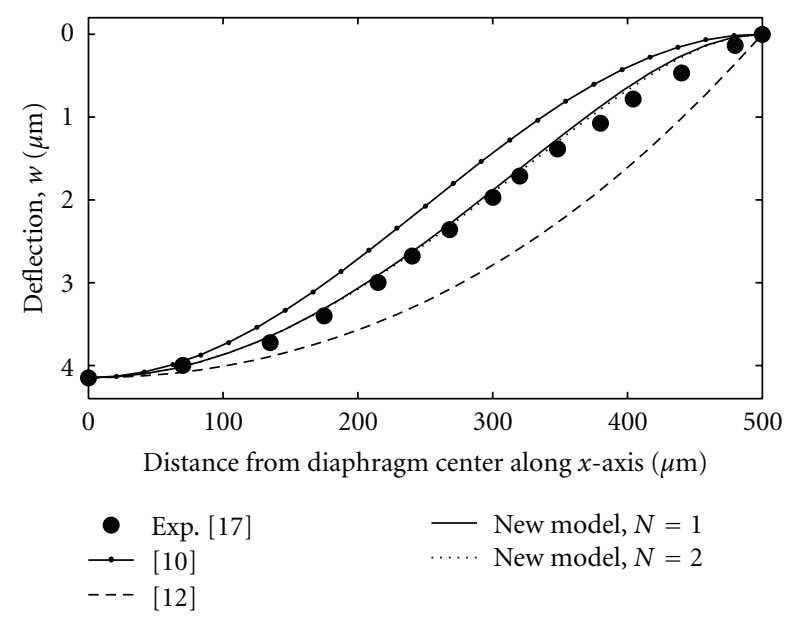

Figure 18: Comparison of FEA deflection profile with the new and the existing models for a $13-\mu \mathrm{m}$ thick single crystal silicon diaphragm presented in [17], plotted from center to diaphragm edge.

acoustic holes, the actual capacitance between the diaphragm and the backplate would be lower than the capacitance if there were no holes in the diaphragm. Therefore, to account for this fact, capacitance between the diaphragm and the backplate was calculated first assuming no holes, and then an equivalent amount of capacitance equal to the area of the acoustic holes was subtracted to obtain the total capacitance. With this procedure, a capacitance value of $2.13 \mathrm{pF}$ for the undeflected diaphragm is obtained, which is very close to the reported measured value of $2.12 \mathrm{pF}$ [18], showing an accuracy better than $0.5 \%$. The capacitance values calculated using the new deflection shape function (11) show very good agreement with the experimental results with a maximum deviation of $1.7 \%$ in the measurement range.

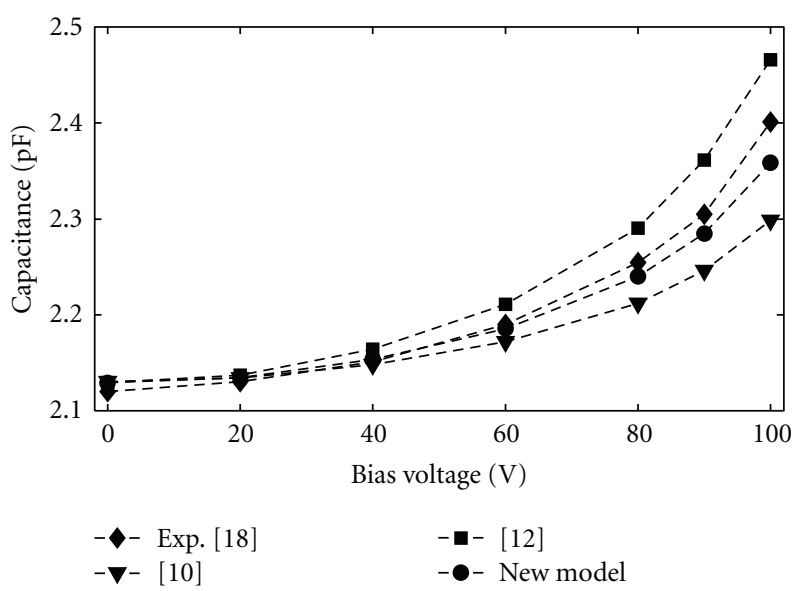

FIgURE 19: Comparison of capacitance values calculated using the developed model with the experimental results of a MEMS capacitive microphone, measured at different bias voltages, presented in [18]. The capacitance values are calculated for the three deflection shape functions following $[10,12]$ and this model (11). The microphone consists of a $3-\mu \mathrm{m}$ thick Al diaphragm, size $0.5 \mathrm{~mm} \times$ $0.5 \mathrm{~mm}$, with acoustic holes separated from the rigid backplate with an airgap of about $1 \mu \mathrm{m}$.

\section{Conclusions}

A highly accurate analytical model to calculate the capacitance of CMUT devices with clamped square diaphragm subject to a uniform pressure loading has been presented. A new deflection shape function is introduced to more accurately calculate the deformation curves of square diaphragms, and fringing field effect is incorporated in the developed capacitance model. The new deflection shape function is simple, easy to use, and applicable to both small and large diaphragm deflections. The new model predicted deflection profiles are in excellent agreement with both the experimental and IntelliSuite FEA results. The developed model is used to calculate the capacitance between a deformed thinsquare diaphragm and a fixed backplate in a typical CMUT device for a wide range of geometry, material properties, and loading conditions. The capacitance values are found to be in excellent agreement with both the experimental and $3 \mathrm{D}$ electromechanical FEA carried out using IntelliSuite with a maximum deviation of about $1.7 \%$ in contrast to the $16-$ $19 \%$ deviations if models available elsewhere are used. The model can help in improving the design methodology of CMUTs and can be extended to design other MEMS-based transducers where pressure-loaded square diaphragms are used.

\section{Acknowledgments}

This research has been supported by the Natural Sciences and Engineering Research Council of Canada (NSERC) Discovery Grant no. RGPIN 293218 and Ontario Centres of Excellence (OCE) Grant no. IC50659. The authors would like to greatly acknowledge the additional generous support provided by the Canadian Microelectronics Corporation 
(CMC Microsystems), the IntelliSense Software Corporation of Woburn, MA, USA.

\section{References}

[1] Integrated Sensing Systems, Inc. (ISSYS), "Technology: Capacitive Sensing," http://www.mems-issys.com/capacitive .shtml.

[2] O. Oralkan, A. S. Ergun, J. A. Johnson et al., "Capacitive micromachined ultrasonic transducers: next-generation arrays for acoustic imaging," IEEE Transactions on Ultrasonics, Ferroelectrics and Frequency Control, vol. 49, no. 11, pp. 15961610, 2002.

[3] M. Meloche and S. Chowdhury, "Design of a MEMS discretized hyperbolic paraboloid geometry ultrasonic sensor microarray," IEEE Transactions on Ultrasonics, Ferroelectrics and Frequency Control, vol. 55, no. 6, pp. 1363-1372, 2008.

[4] R. Puers and D. Lapadatu, "Electrostatic forces and their effects on capacitive mechanical sensors," Sensors and Actuators $A$, vol. 56, no. 3, pp. 203-210, 1996.

[5] S. Timoshenko and S. Woinowsky-Krieger, Theory of Plates and Shells, McGraw-Hill, New York, NY, USA, 2nd edition, 1959.

[6] N. Ben Moussa, Conception, modélisation et réalisation d'un capteur de pression capacitif micro électronique, Ph.D. thesis, LAAS, Toulouse, France, 1985.

[7] F. Kerrour and F. Hobar, "A novel numerical approach for the modeling of the square shaped silicon membrane," Semiconductor Physics, Quantum Electronics and Optoelectronics, vol. 9, no. 4, pp. 52-57, 2006.

[8] W. Stewart, "Uniformly loaded, clamped, rectangular plates with large deflection," in Proceedings of the 5th International Congress for Applied Mechanics (Cambridge, Mass, USA, 1938), pp. 123-128, John Wiley \& Sons, 1939.

[9] S. Levy, "Square plate with clamped edges under normal pressure producing large deflections," Tech. Rep. 740, 1942.

[10] H. E. Elgamel, "Closed-form expression of the relationships between stress, membrane deflection, and resistance change with pressure in silicon piezoresistive pressure sensors," Sensors and Actuators A, vol. 50, no. 1-2, pp. 17-22, 1995.

[11] X. Y. Ye, J. H. Zhang, Z. Y. Zhou, and Y. Yang, "Measurement of young's modulus and residual stress of micromembranes," in Proceedings of the 7th International Symposium on Micro Machine and Human Science, pp. 125-130, Nagoya Municipal Industrial Research Institute, Nagoya, Japan, October 1996.

[12] D. Maier-Schneider, J. Maibach, and E. Obermeier, "A new analytical solution for the load-deflection of square membranes," Journal of Microelectromechanical Systems, vol. 4, no. 4, pp. 238-241, 1995.

[13] S. D. Senturia, Microsystems Design, Kluwer Academic, Norwell, Mass, USA, 2000.

[14] H. E. A. Elgamel, "A simple and efficient technique for the simulation of capacitive pressure transducers," Sensors and Actuators A, vol. 77, no. 3, pp. 183-186, 1999.

[15] N. V. D. Meijs and J. T. Fokkema, "VLSI circuit reconstruction from mask topology," Integration, vol. 2, no. 2, pp. 85-119, 1984.

[16] K. Nabors, T. Korsmeyer, and J. White, "Multiple accelerated preconditioned iterativemethods for solving threedimensional mixed first and second kind integral equations," http://www.rle.mit.edu/cpg/publications/pub106.pdf.

[17] T. Pancewicz, R. Jachowicz, Z. Gniazdowski, Z. Azgin, and P. Kowalski, "The empirical verification of the FEM model of semiconductor pressure sensor," Sensors and Actuators A, vol. 76, pp. 260-265, 1999.

[18] B. A. Ganji and B. Y. Majlis, "Design and fabrication of a new MEMS capacitive microphone using a perforated aluminum diaphragm," Sensors and Actuators A, vol. 149, no. 1, pp. 29$37,2009$. 

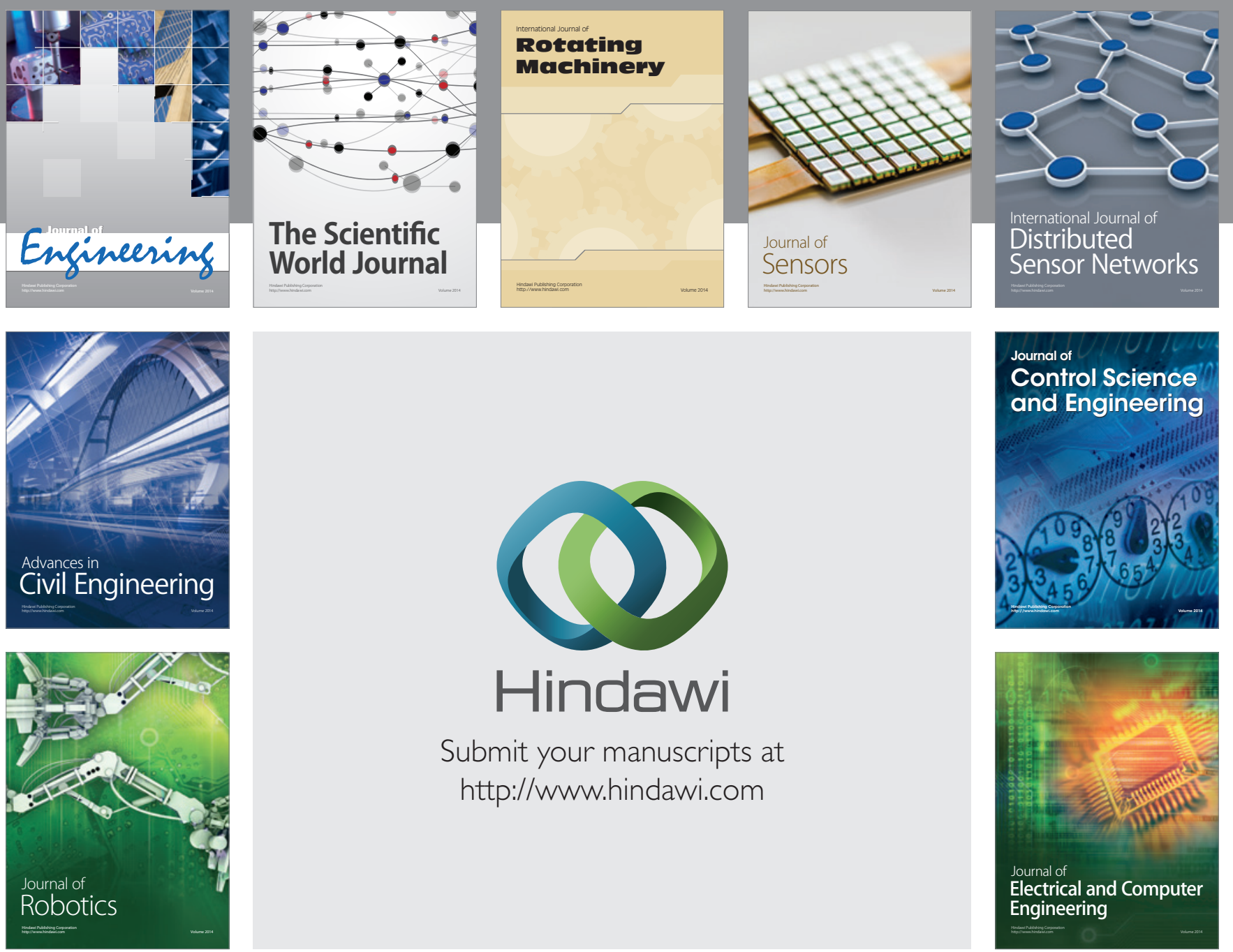

Submit your manuscripts at

http://www.hindawi.com
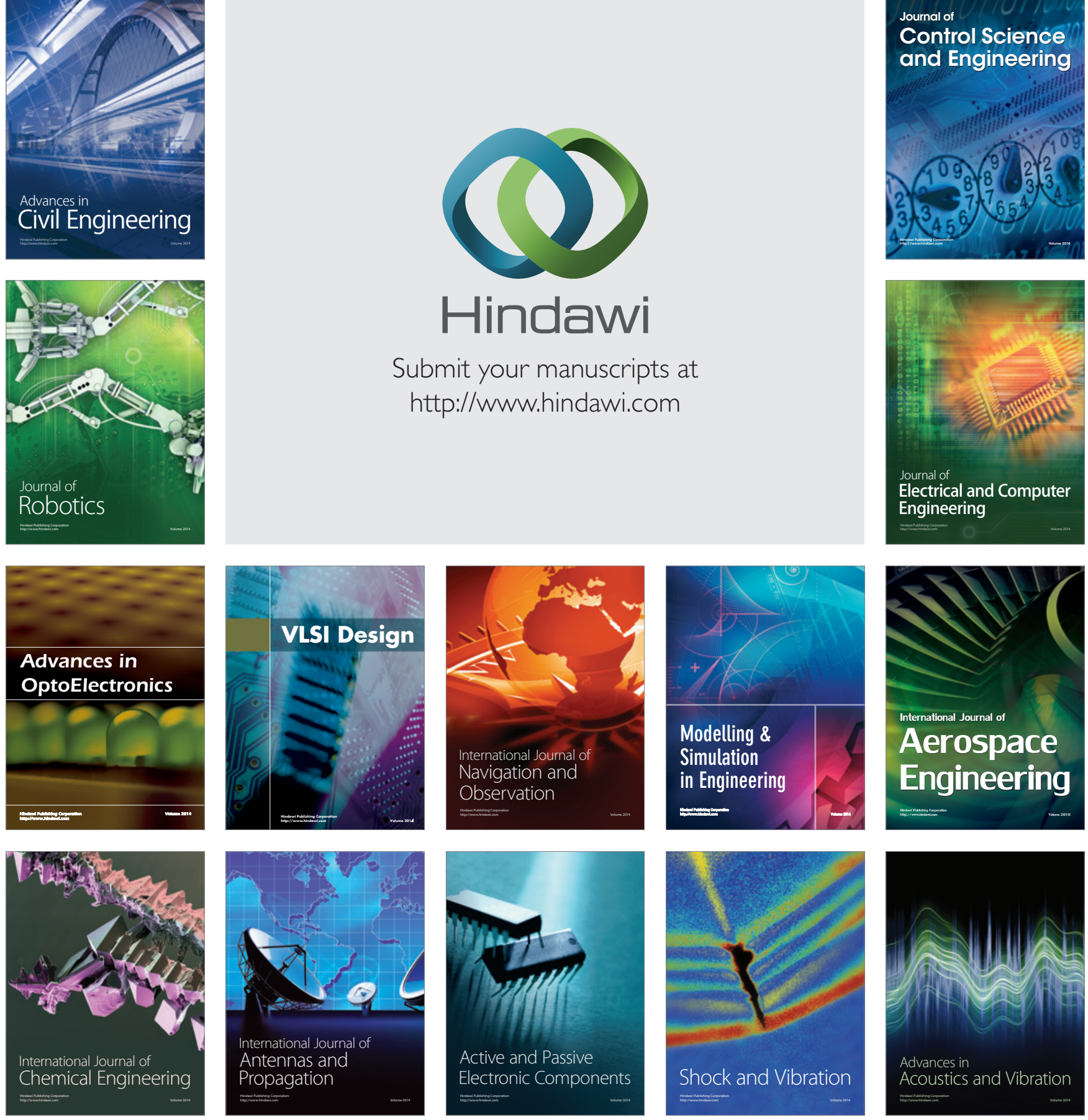